\title{
OPTICAL REFLECTANCE IN FIBROUS TISSUES AND \\ SKELETAL MUSCLES
}

Janaka C. Ranasinghesagara

Dr. Gang Yao, Dissertation Supervisor

\begin{abstract}
We studied diffuse optical reflectance in two anisotropic biological tissues: high moisture soy extrudates and skeletal muscles. High moisture extrusion of soy proteins can produce healthy alternative protein sources that resemble real meat. Quantitative assessment of fiber formation in such meat analogs is an important quality control in the manufacturing process. We studied light propagation in soy extrudates and developed a non-destructive method to measure fiber formation. The method was successfully implemented in a fast laser scanning system for real time measurement. In addition to fibrous structures, skeletal muscle has a unique repetitive microstructure which plays an important role in light-muscle interaction. We developed an optical model to study the effects of various myofibril morphologies in laser diffraction by single muscle fibers. By incorporating such diffraction effect into a Monte Carlo model, we reproduced the unique reflectance patterns observed in whole skeletal muscles. Our study on 14 steers showed the profiles of diffuse reflectance are subject to the effects of animal breed, muscle type and aging. Overall, optical measurements are promising for characterizing beef samples.
\end{abstract}

